\title{
Characterization of Shape Memory Polymer Estane by Means of Dynamic Mechanical Thermal Analysis Technique
}

\author{
Rasa Kazakevičiūtė-Makovska, Aycan Özlem Özarmut, and Holger Steeb \\ Mechanics-Continuum Mechanics, Ruhr University Bochum, Universitätsstraße 150, 44780 Bochum, Germany \\ Correspondence should be addressed to Rasa Kazakevičiūtè-Makovska; rasa.kazakeviciute-makovska@rub.de
}

Received 27 September 2013; Accepted 1 December 2013; Published 9 January 2014

Academic Editor: Chris Bowen

Copyright (C) 2014 Rasa Kazakevičiūtè-Makovska et al. This is an open access article distributed under the Creative Commons Attribution License, which permits unrestricted use, distribution, and reproduction in any medium, provided the original work is properly cited.

\begin{abstract}
Commercially available shape memory polymer (SMP) Estane (designation: ETE75DT3 NAT022) is investigated by means of dynamic mechanical thermal analysis (DMTA) technique in torsion mode using the Modular Compact Rheometer MCR-301 (Anton Paar $\mathrm{GmbH}$ ). Amplitude sweep tests have been run below and above the glass transition temperature to establish the linear viscoelastic range (LVR) in glassy and rubbery phase of this SMP for the correct physical interpretation of DMTA data. Temperature sweep tests were performed at various frequencies to study the influence of this parameter on values of the storage and loss moduli and the storage and loss compliances as well as the viscosities. These tests have been carried out in heating mode with different rates and at different strain amplitudes. The short- and long-term behavior of SMP Estane have been studied by frequency sweep tests performed at different temperatures and data have been transformed into time-domain properties by applying time-temperature superposition principles. All these DMTA data provide the experimental basis for the study of relaxation processes, propertystructure relationships, and the shape memory effect in this little-known SMP.
\end{abstract}

\section{Introduction}

Thermoresponsive shape memory polymers (SMPs) have the ability to recover a permanent shape from a programmed temporary shape upon heating. The characterization and modeling of this phenomenon, known as the shape memory effect (SME), require comprehensive experimental studies of mechanical, thermal, and functional properties of this class of smart materials [1-12]. In particular, the temperatureand time-dependent behavior of thermoresponsive SMPs are one of the most important indicators of their functional properties such as shape fixity, shape recovery, and stress recovery $[2,6,10,12]$. Moreover, the shape recovery property is directly related to the temperature and frequency (time) dependency of elastic and viscoelastic material parameters, which must be determined using reliable measuring methods for every particular SMP.

The viscoelastic behavior of polymers including SMPs may be studied in several experimental methods like steadystate deformation, stress relaxation, creep, or oscillatory dynamic deformation. The results of tests are quantified using material functions such as steady viscosity, relaxation modulus, creep compliance, and storage and loss modulus. Additional tests are needed to study the coupling between viscoelastic and thermal properties of polymers. The standard quasi-static tensile tests together with creep and stress relaxation tests are often carried out at different ambient temperatures to determine the temperature-dependent mechanical response of the particular polymer under monotonic and steady-state loading $[13,14]$. Such tests, although straightforward and reliable, are costly in both materials and time and often limited to selected temperatures, short-term responses, and low strain rates.

Dynamic mechanical thermal analysis (DMTA) is a very efficient alternative technique for the study of time-, frequency-, and temperature-dependent mechanical properties of polymeric materials $[14,15]$ and may be used to characterize various polymers including thermoplastics, thermosets, elastomers, and polymer blends as well as SMPs. This technique provides also information about relaxation 
processes in polymers, specifically, the glass transition and subglass processes [14-16]. This makes DMTA particularly useful for the characterization of SMPs and it becomes an indispensable experimental method in study of these smart materials. Additional advantages of using DMTA to characterize the thermoviscoelastic behavior of SMPs include automated testing, precise control of the test environment, simple preparation of test specimens, and possibility of performing tests in a wide range of temperatures.

In this work, we present results of an extensive DMTA study of the commercially available thermoplastic polyurethane (TPU) based SMP Estane (purchased from Lubrizol, Oevel Westerlo, Belgium) performed in torsion deformation mode using the Modular Compact Rheometer MCR-301 equipped with temperature chamber. The DMTA investigations include the following specific experiments:

(i) strain amplitude sweep tests at different temperatures to determine the linear viscoelastic range (LVR) for the tested polymer,

(ii) temperature sweep tests at different frequencies with the aim to study the coupling between temperature and time-dependent properties and to evaluate relaxation processes,

(iii) temperature sweep tests in heating mode with different rates to evaluate the influence of this parameter on viscoelastic properties,

(iv) temperature sweep tests on samples cut out from plates in two perpendicular directions to identify a possible anisotropy in material structure or processing of the tested polymer,

(v) frequency sweep tests at different isothermal temperatures to determine the short- and long-time response of this SMP.

These special tests serve to characterize various aspects and relative contributions of viscous and elastic responses of the Estane. In particular, the frequency of oscillation defines the timescale of tests and it follows that by observing polymer response as a function of frequency, the material can be probed at different timescales. These measurements are important in SMPs characterization because the overall response of these materials is due to contribution from several mechanisms at the molecular and microscopic levels. These mechanisms can be identified by observing material response at different frequencies.

Conventional DMTA equipments such as the one used in this study provide data for a tested SMP in a limited temperature and frequency range. However, when combined with theoretical concepts generally known as superposition principles and related concepts of the so-called master curves, these data may be used to determine the viscoelastic behavior of the same material over a wider frequency (or time) range. The application of these theoretical concepts to SMP Estane and the representative results are presented in the second part of the paper. Finally, the experimental and analytical (theoretical) results for the tested polymer obtained in this study are shortly discussed in reference to other thermoresponsive SMPs extensively investigated in the literature.
The SMP Estane has not yet drawn much attention on its thermoviscoelastic properties. The only known study is [17], where the material stability, the functional fatigue, and some thermal properties of this SMP have been investigated. The DMTA results presented in this work provide complementary data for this new smart polymer.

\section{Experimental}

2.1. Material. The thermoplastic polyurethane-based shape memory polymer Estane (designation: ETE75DT3 NAT022) was purchased from Lubrizol (Oevel Westerlo, Belgium) in the form of plates of dimensions $80 \times 90 \times 2 \mathrm{~mm}^{3}$. The plates were cut into rectangle shape specimens with dimensions of $10 \times 50 \times 2 \mathrm{~mm}^{3}$ (Figure 1).

The same SMP has been previously studied in [17] using specimens that were injection-molded from granulates also purchased from Lubrizol.

2.2. Experimental Setup and Test Procedures. Dynamic mechanical thermal analysis (DMTA) tests in torsion deformation mode were performed in the temperature range from $-5^{\circ} \mathrm{C}$ to $150^{\circ} \mathrm{C}$ using the Modular Compact Rheometer MCR301 (Anton Paar $\mathrm{GmbH}$ ) [18] equipped with standard fixtures (SRF12) for rectangular bars and a temperature chamber (CDT-180) having high temperature stability $\left( \pm 0.3^{\circ} \mathrm{C}\right)$. The equipment and details of a fixed specimen are shown in Figure 2.

In DMTA torsion mode tests, a small axial force (around $-0.5 \mathrm{~N}$ ) is applied to the sample in order to maintain it under net tension. On this state of sample, the harmonic twist angle (rotation) with prescribed amplitude and frequency is superimposed and the resulting harmonic torque as well as the phase lag or loss angle $\delta$ (in rad) is measured. From these raw data and sample dimensions, the RheoPlus Software [19] computes the corresponding shear stress $\tau$ and shear strain $\gamma$, which in turn are used to determine the dynamic shear modulus $\left|G^{*}\right|$, the shear storage and loss moduli, $G^{\prime}$ and $G^{\prime \prime}$, respectively, and the loss factor $\lambda$. These material parameters are related by the following formulae $[14,15]$ :

$$
\begin{gathered}
\left|G^{*}\right|=\sqrt{G^{\prime 2}+G^{\prime \prime 2}}, \quad G^{\prime}=\left|G^{*}\right| \cos \delta, \\
G^{\prime \prime}=\left|G^{*}\right| \sin \delta, \quad \lambda=\tan \delta=\frac{G^{\prime \prime}}{G^{\prime}} .
\end{gathered}
$$

The complex modulus, $G^{*}=G^{\prime}+i G^{\prime \prime}, i^{2}=-1$, determined in the strain-controlled DMA tests describes the relaxation of the mechanical stress for a given strain. In the stresscontrolled DMA experiment, a stress is given and the resulting strain is measured. In this case, the complex shear compliance $J^{*}$ is determined directly from the applied stress and measured strain. Equivalently, it may be computed from the shear moduli measured in strain-controlled tests using the following theoretical formulae $[14,15]$ :

$$
\left|J^{*}\right|=\frac{1}{\left|G^{*}\right|}, \quad J^{\prime}=\frac{G^{\prime}}{\left|G^{*}\right|^{2}}, \quad J^{\prime \prime}=\frac{G^{\prime \prime}}{\left|G^{*}\right|^{2}},
$$




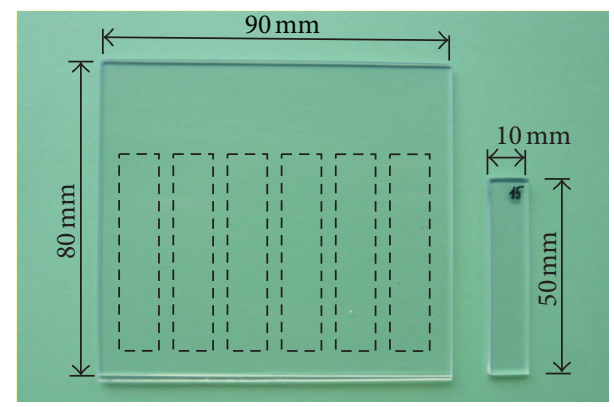

FIGURE 1: Rectangular specimens cut out of the SMP Estane plate.

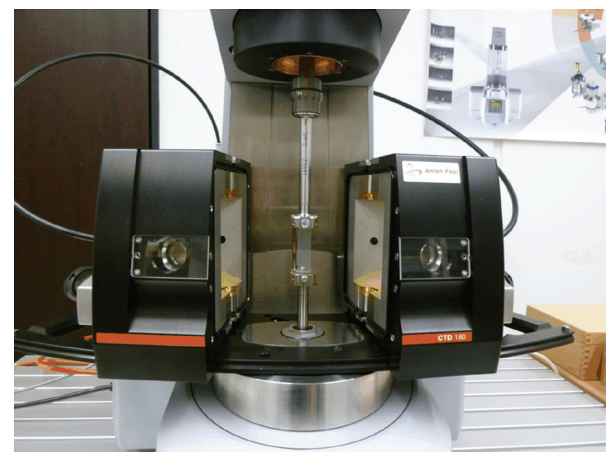

(a)

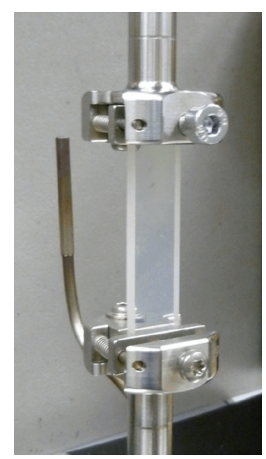

(b)

FIgUre 2: Modular Compact Rheometer MCR-301 equipped with temperature chamber (a) and details of specimen clamping (b).

where $J^{\prime}$ and $J^{\prime \prime}$ are the storage and loss compliance, respectively. The complex compliance $J^{*}$ obtained in the stresscontrolled DMA tests, which is simply the inverse or reciprocal of the complex shear modulus, describes the strain retardation, and the retardation time is a measure of the time delay in strain after imposition of the stress.

Besides the shear modulus and the shear compliance, there is another quantity called the viscosity to characterize the rheological behavior of polymeric materials. The complex viscosity is defined as the ratio of the stress and strain rate and may be computed from the complex shear modulus $G^{*}$ and the frequency $\omega[14,15]$ :

$$
\left|\eta^{*}\right|=\frac{\left|G^{*}\right|}{\omega}, \quad \eta^{\prime}=\frac{G^{\prime \prime}}{\omega}, \quad \eta^{\prime \prime}=\frac{G^{\prime}}{\omega},
$$

where $\eta^{\prime}$ and $\eta^{\prime \prime}$ are the dynamic and out-of-phase viscosities, respectively. The physical significance of all these dynamic quantities measured in DMTA may be better appreciated in terms of energy stored and dissipated during harmonic deformation $[14,15]$.

\section{Results and Data Analysis}

The rheometer used in this study can perform a wide range of DMTA experiments including temperature ramp, frequency, and amplitude sweep tests in both stress- and strain-controlled modes. From such tests, the determined shear moduli and the loss factor are obtained as functions of test parameters in the specified range.

3.1. Strain Amplitude Sweep Data. In order to use DMTA technique to accurately determine thermorheological properties and to develop morphological relationships of materials, a tested polymer must be deformed at amplitudes that remain within the linear viscoelastic region (LVR). Within LVR, the viscoelastic response of the polymer is independent of the magnitude of deformation. As a general rule, this region must be determined for every type of polymer by DMTA amplitude sweep tests, in which a frequency is fixed and the strain amplitude is incrementally increased.

From the plot of the storage and loss moduli against the strain amplitude for the SMP Estane shown in Figure 3, the linear viscoelastic region is read off for three temperatures: the room temperature and temperatures well-below and wellabove the glass transition temperature. It is seen that for the shear strain amplitude up to $2 \%$, the shear moduli remain nearly constant and this determines the LVR for the Estane. The strain sweep test is the first step in dynamic mechanical analysis and is always performed prior to a frequency sweep test in order to determine an appropriate strain level for temperature and frequency sweeps.

3.2. Temperature Sweep Data. The temperature-sweep test involves measurements of the storage and loss moduli and the loss factor over a specified temperature range at constant strain (or stress) amplitude and constant frequency. Temperature sweeps can be carried out in ramp or stepwise fashion.

Figures 4-6 show DMTA data obtained in the temperature ramp tests, which were performed under the straincontrolled mode at seven frequencies (indicated in the figures) with the shear strain amplitude of $0.01 \%$ and the heating rate $1 \mathrm{~K} / \mathrm{min}$.

In Figure 4(a), the storage shear modulus $G^{\prime}$ and the loss shear modulus $G^{\prime \prime}$ are plotted against the temperature from $-5^{\circ}$ to $150^{\circ} \mathrm{C}$. It is seen that the storage modulus $G^{\prime}$ decreases gradually with increasing temperature until attaining the region of the glass transition. Starting from that region, changes in $G^{\prime}$ with temperature are remarkably different for low and high frequencies. For the frequencies higher than $2 \mathrm{~Hz}$, a more rapid reduction in values of the storage modulus occurs. However, for the frequencies smaller than $2 \mathrm{~Hz}$, nonmonotonic variations of $G^{\prime}$ with temperature are observed. The loss modulus $G^{\prime \prime}$ at all test frequencies 


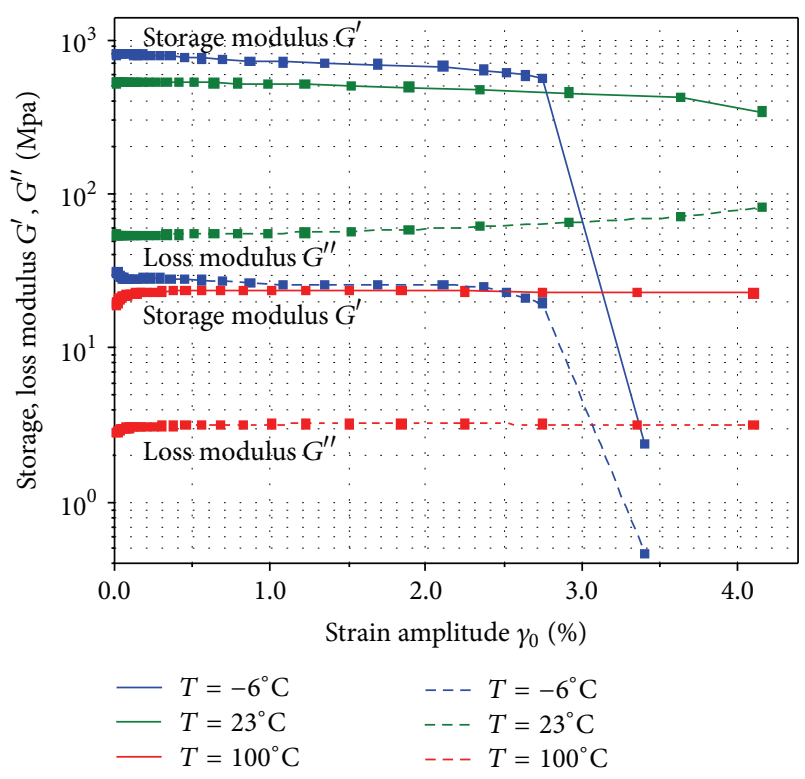

FIgURE 3: Amplitude sweep test at different temperatures: variation of the storage and loss shear moduli with strain amplitude.

shows a nonmonotonic variation with temperature and this distinguishes the Estane from other investigated SMPs [8, 9, 20].

Typically, the DMA temperature sweep tests of a polymer sample scanned at different frequencies show that at higher frequencies the storage modulus demonstrates higher values and the glass transition temperature shifts to a higher temperature. Figure 4(b) shows variations in the loss factor (damping coefficient) with temperature for all tested frequencies. The temperature at which this coefficient reaches its maximum value is interpreted as the glass transition temperature of material $[14,15]$. It is seen in Figure 4(b) that both the maximum value of the loss factor and the glass transition temperature increase with the increase of test frequency.

DMTA measurements over a range of temperatures provide valuable insight into the structure, the morphology, and the viscoelastic behavior of SMPs. In particular, these measurements are an important part of the technique for establishing relaxation transitions. For example, during temperature sweep, the temperature at crossover modulus $G^{\prime}=$ $G^{\prime \prime}$ is considered to signify the beginning of gel forming (or gel melting) temperature. During polymer melting, the temperature at crossover modulus is an indication of the "softening point" of the polymer, the onset temperature of rapid melt and flow. The temperature sweep test is also helpful to detect changes that would occur at rather high, and possibly inaccessible, frequencies if measurements were made at room temperature.

A further characterization of the tested SMP is obtained by plotting the storage and loss compliances as well as the dynamic and out-of-phase viscosities as functions of temperature for different frequencies (Figures 5 and 6). All these material parameters show the nonmonotonic behavior. It is

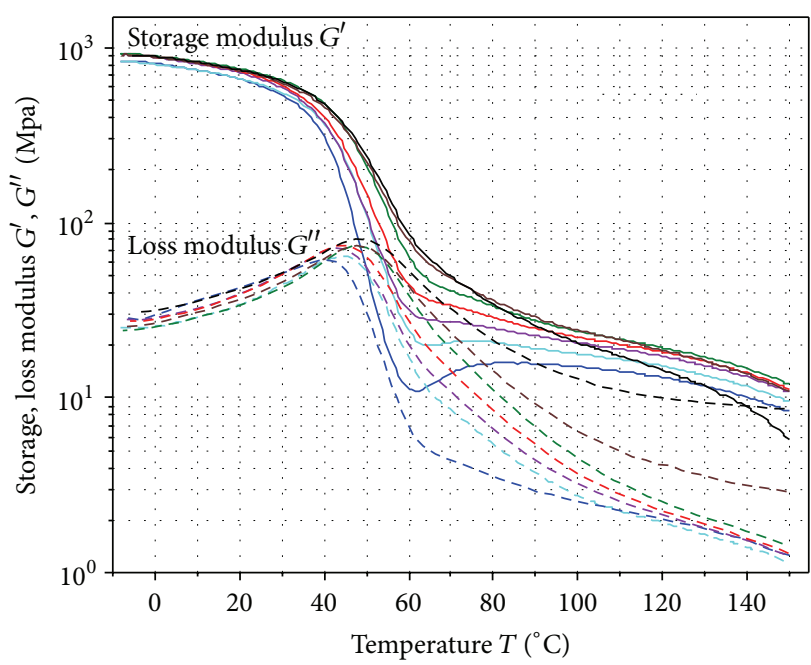

Frequency
$f=0.1 \mathrm{~Hz}$
$f=1.0 \mathrm{~Hz}$
$f=2 \mathrm{~Hz}$
$f=5 \mathrm{~Hz}$
$\begin{aligned} f & =10 \mathrm{~Hz} \\ f & =20 \mathrm{~Hz}\end{aligned}$
$f=30 \mathrm{~Hz}$

(a)

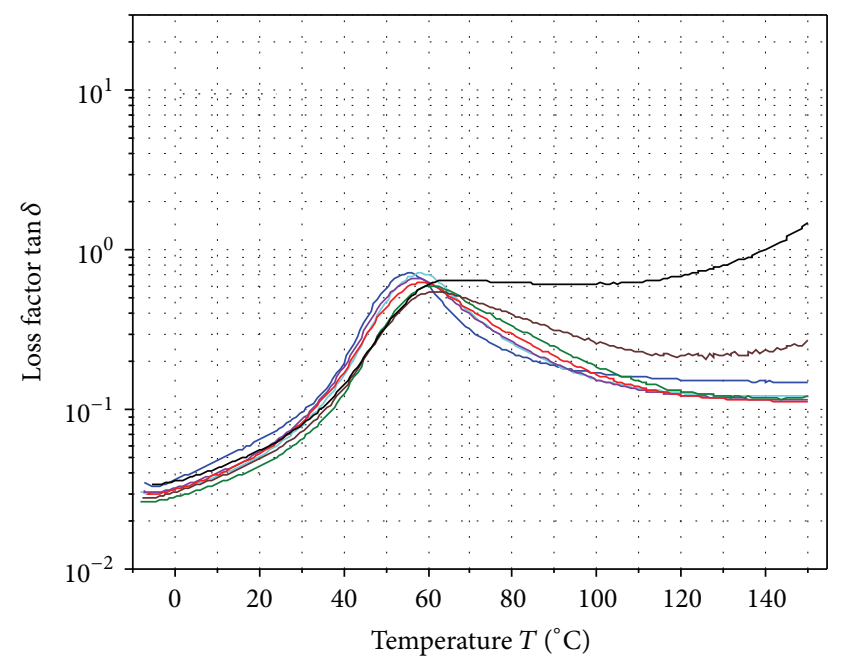

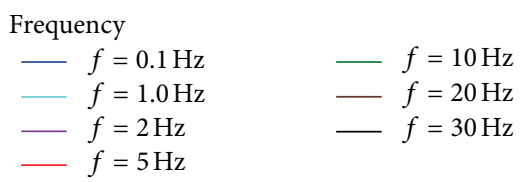

(b)

FIGURE 4: Temperature scan at different frequencies: variation of the storage and loss shear moduli (a) and loss factor (b) with temperature.

also seen that the viscosities display a stronger dependency on frequency than the moduli and the compliances.

3.3. Heating Rate Effects. Figure 7(a) shows a plot of the storage and loss moduli as functions of temperature for different heating rates at constant testing frequency $(1 \mathrm{~Hz})$ and constant strain amplitude $(0.01 \%)$. The purpose of such 


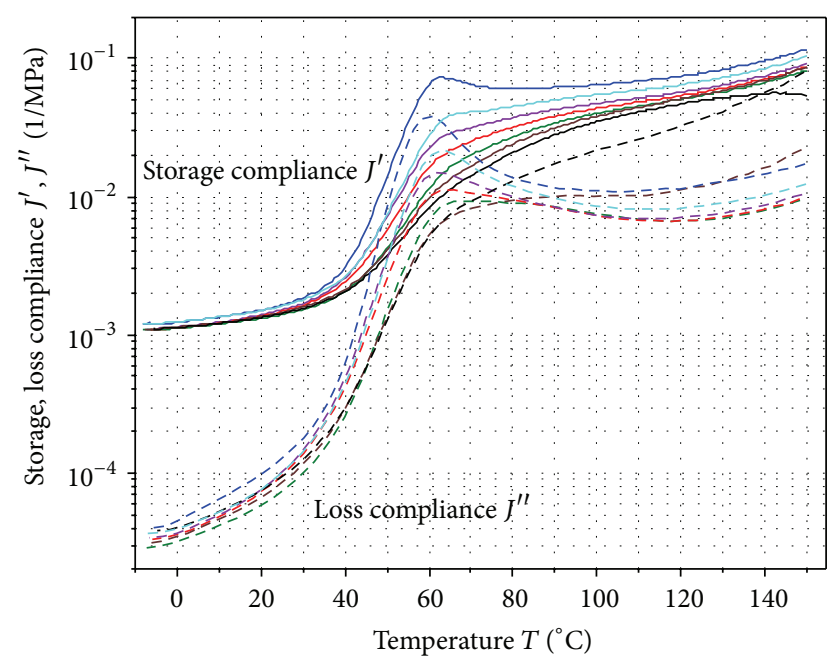

Frequency
$-f=0.1 \mathrm{~Hz}$
$f=1.0 \mathrm{~Hz}$
$f=10 \mathrm{~Hz}$
$-f=2 \mathrm{~Hz}$
$f=5 \mathrm{~Hz}$

Figure 5: Temperature scan at different frequencies: variation of the storage and loss compliances with temperature.

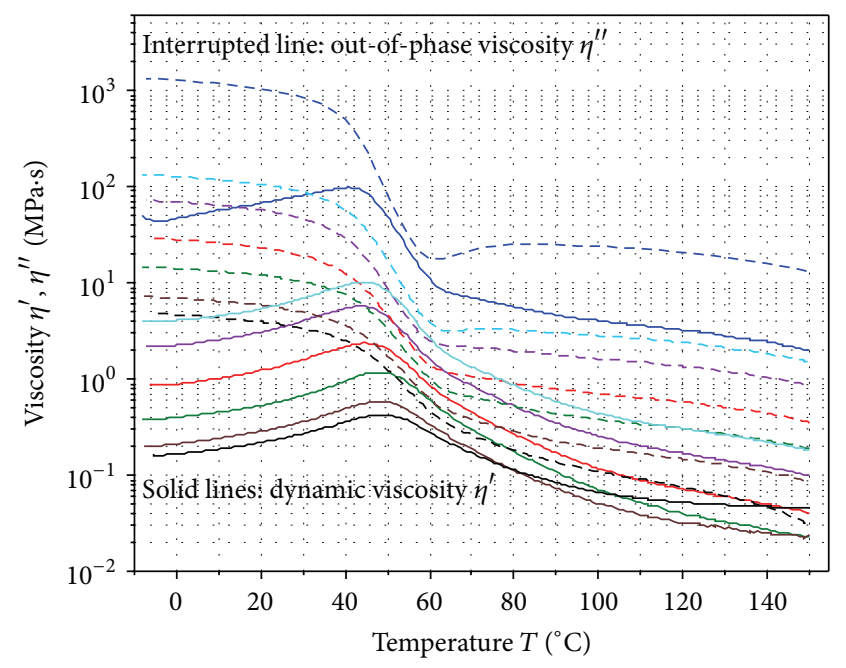

$$
\begin{array}{rlrl}
\text { Frequency } & & \\
-f & =0.1 \mathrm{~Hz} & -f & =10 \mathrm{~Hz} \\
f & =1.0 \mathrm{~Hz} & -f & =20 \mathrm{~Hz} \\
f & =2 \mathrm{~Hz} & -f & =30 \mathrm{~Hz} \\
f & =5 \mathrm{~Hz} & &
\end{array}
$$

Figure 6: Temperature scan at different frequencies: variation of the dynamic and out-of-phase viscosities with temperature.

tests is to examine effects of heating rate on the transition behavior and other processes in polymeric material. It is seen that within experimental error the transition behavior does not appreciably alter with change in heating rate in the tested range.

The loss factor results are shown in Figure 7(b). The peaks are in this case very well resolved, and the temperatures of maxima are easily detected. A small shift towards higher

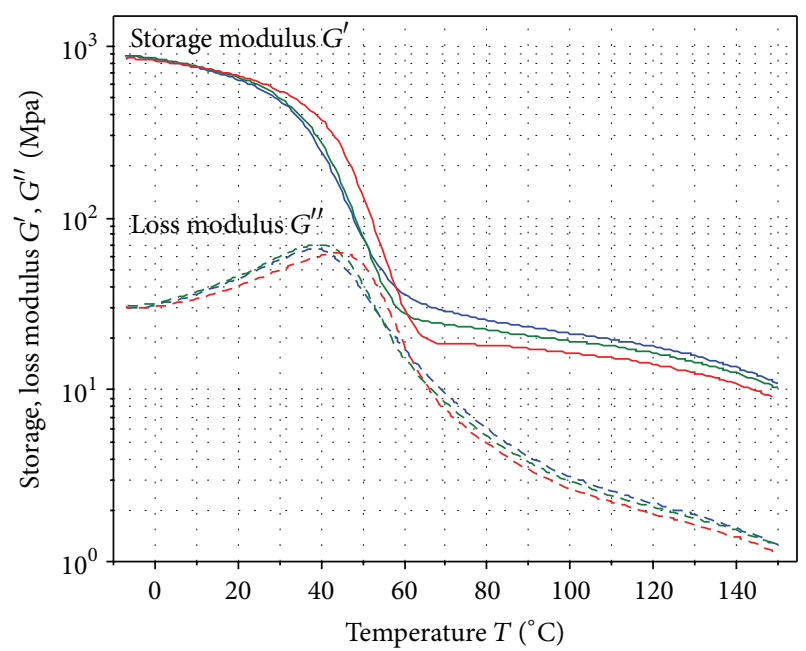

Heating rate

$-\beta=0.5^{\circ} \mathrm{C} / \mathrm{min}$

$-\beta=1.0^{\circ} \mathrm{C} / \mathrm{min}$

$-\beta=2.0^{\circ} \mathrm{C} / \mathrm{min}$

(a)

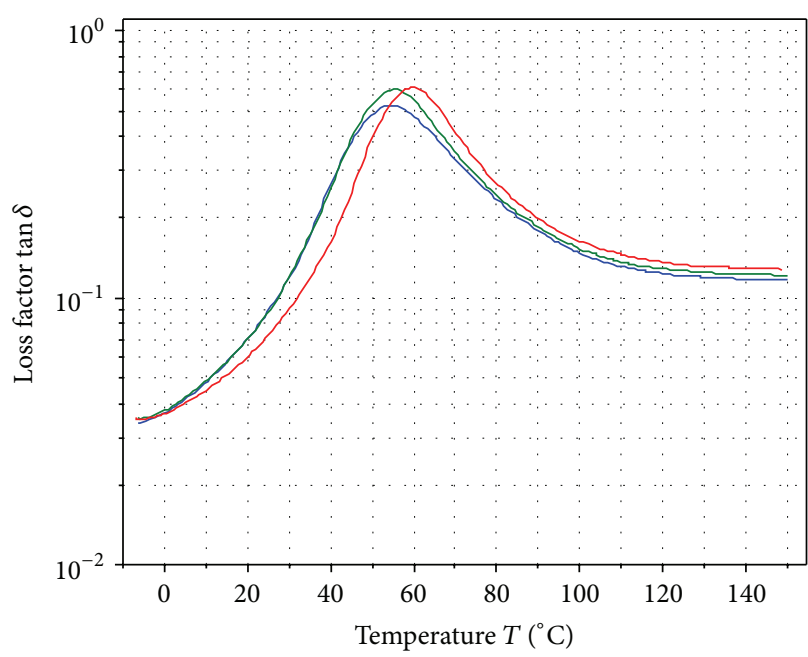

Heating rate

$\begin{aligned}-\beta & =0.5^{\circ} \mathrm{C} / \mathrm{min} \\ \beta & =1.0^{\circ} \mathrm{C} / \mathrm{min} \\ \beta & =2.0^{\circ} \mathrm{C} / \mathrm{min}\end{aligned}$

(b)

FIgURE 7: Temperature scan at different heating rates: variation of the storage and loss shear moduli (a) and the loss factor (b) with temperature.

temperatures is observed for the $\tan \delta$ peak with increasing heating rate. A similar behavior of polymeric materials is usually seen in other thermal analysis techniques in heating mode such as DSC.

3.4. Processing Anisotropy Effects. Only limited research has focused on possible nonisotropic effects in polymeric materials. Such effects could be due to material anisotropy, processing anisotropy, or deformation. Each type of anisotropy greatly complicates the interpretation of DMTA data. The 
analysis of the structural anisotropy in oriented semicrystalline polymers presented in [21] is a good example.

Both the material and processing-induced anisotropy in polymers may be detected by DMTA technique. In [22], SMP Tecoflex has been studied by temperature sweep tests performed on samples cut out in different directions from the injection-molded plates, but no anisotropic effect has been observed. Similar DMTA tests have also been carried out on the polymer Estane and the representative results are shown in Figure 8. The tested polymer has been purchased from Lubrizol in the form of plates (Figure 1), but the processing details are unknown. Nevertheless, it is seen (Figure 8) that the temperature sweep on the samples cut out from the plate in two perpendicular directions shows nearly identical values of the storage and loss moduli. This proves that this polymer is isotropic in respect to its material structure as well as processing.

\section{Long-Term Behavior}

4.1. Frequency Sweep Data. The frequency sweep is probably the most efficient DMTA test in characterizing the viscoelastic behavior of polymeric materials including SMPs. Such a test performed in torsion mode at fixed strain amplitude and temperature provides the storage and loss shear moduli as well as the loss factor as functions of frequency. The corresponding shear compliance and viscosity of a material may then be computed using formulae (2) and (3).

A typical dynamic mechanical analyzer such as that used in this study can provide data only over a limited range of frequency or time and this is inadequate to track the long-term viscoelastic behavior of a tested material. The time-temperature superposition principle not only offers the opportunity to obtain the long-term behavior of polymeric materials from the standard DMTA tests but also provides data that are difficult to measure directly [14, 15]. This principle is based on the empirical assumption that the viscoelastic behavior of a polymer at one temperature is related to the viscoelastic behavior at other temperatures by a shift in frequency or time scale only [23]. In other words, the frequency (or time) and the temperature in viscoelastic data are equivalent, and data at one temperature can be superimposed upon data taken at different temperature merely by shifting the curves. This has been shown to be true for numerous polymeric materials [14, 15, 23-25].

4.2. Superposition Principles and Master Curves. In order to verify the applicability of the time-temperature (equivalently the frequency-temperature) superposition principle for the tested polymer Estane, the frequency sweeps were conducted at different isothermal temperatures ranging from $10^{\circ} \mathrm{C}$ to $75^{\circ} \mathrm{C}$ and stepping every $5^{\circ} \mathrm{C}$ for each sweep step. In all these tests, the same range of frequencies from 0.01 to $15 \mathrm{~Hz}$ at oscillation amplitude of $0.01 \%$ strain has been kept. The data plotted as log-log curves are shown in Figure 9. In this analysis, the lower frequency range was applied due to a better coincidence of the measured moduli and loss factor than at higher frequencies (Figure 4).

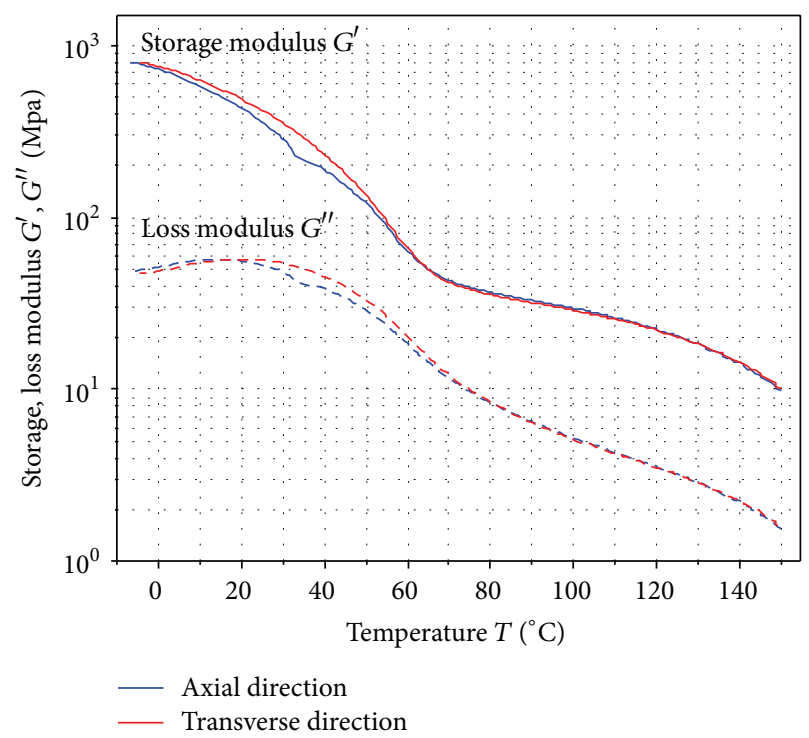

(a)

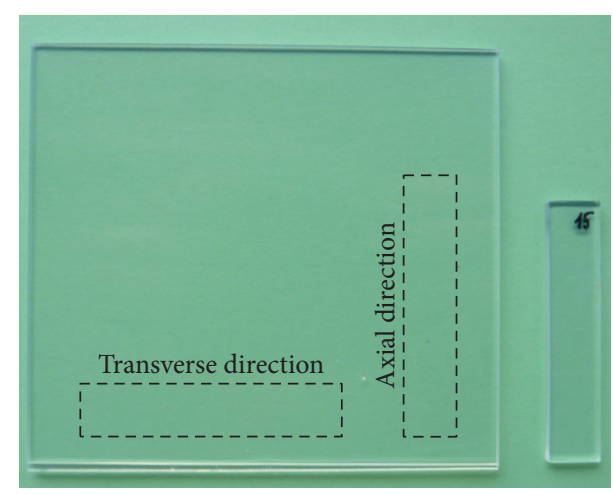

(b)

FIGURE 8: Variation of the storage and loss moduli with temperature (a) measured on samples cut out from the Estane plate in two orthogonal directions (b).

From these data, the master curve has been constructed by shifting some of these curves along the logarithmic frequency axis to the left (to lower frequencies) and others to the right (to higher frequencies) relative to the reference curve at the temperature $T_{0}=50^{\circ} \mathrm{C}$. This temperature is close to the glass transition temperature of Estane $\left(T_{g}=54^{\circ} \mathrm{C}\right)$. The shift factor data were obtained manually from the generation of the storage modulus versus frequency master curve on the log-log scale (see Figure 10). The same shift factor was used to generate the loss modulus versus frequency master curve shown in the same figure.

The materials for which the time-temperature or equivalent principle applies are referred to as thermorheologically simple materials and this may be verified in a number of ways depending on the material parameter used for the study [14, 15]. In the literature [23], the complex modulus has been used to assess the thermorheological simplicity of tested materials. For the Estane, the plot of the master curves (Figure 10) shows that the data at a higher temperature do not superimpose 


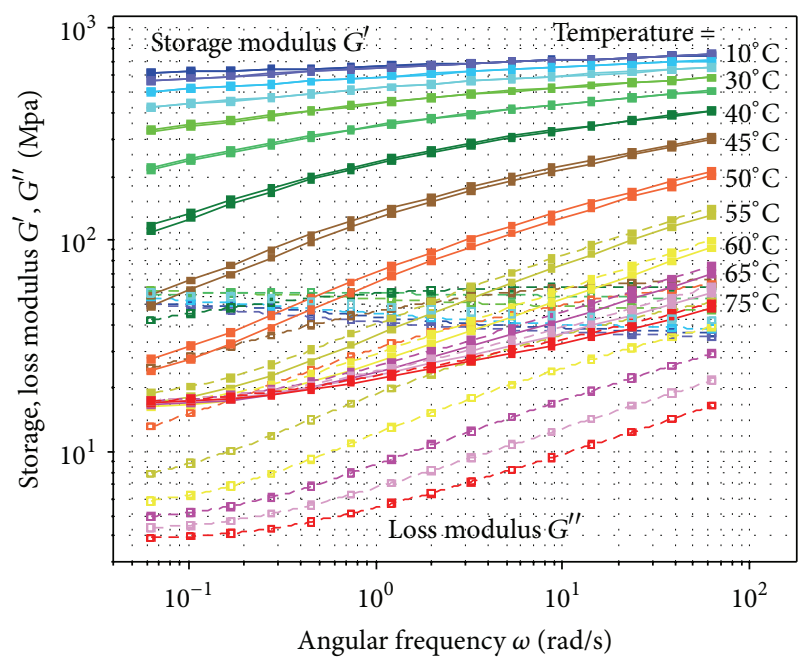

FIGURE 9: Plot of the storage and loss moduli versus frequency (log$\log$ ) for different temperatures.

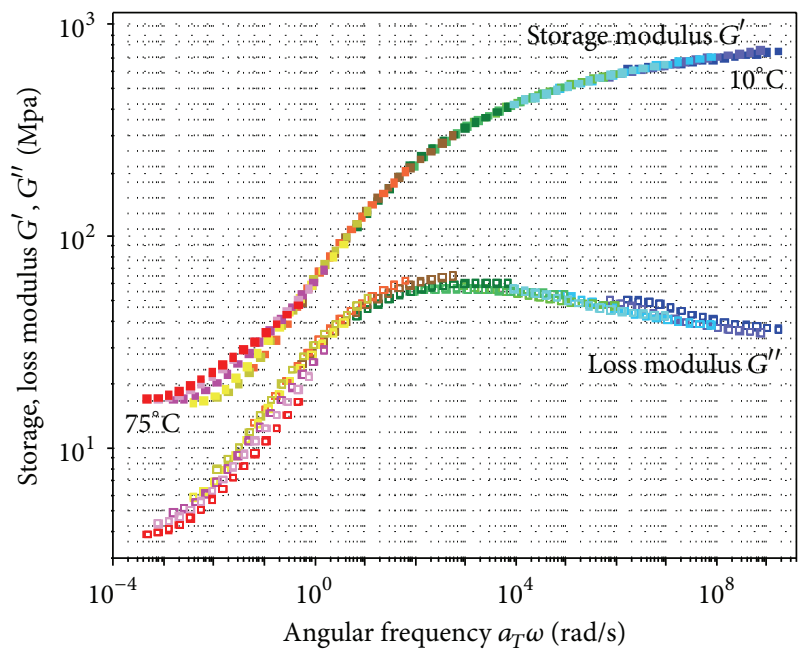

FIGURE 10: The storage and loss moduli versus frequency master curves.

very well; that is, the tested polymer is not in the class of thermorheologically simple materials.

4.3. Determination of Shift Factor Constants. In terms of the dynamic moduli, the time-temperature superposition principle underlying the construction of master curves reads $[14,15,23,26]$

$$
G^{\prime}\left(a_{T} \omega, T\right)=G^{\prime}\left(\omega, T_{0}\right), \quad G^{\prime \prime}\left(a_{T} \omega, T\right)=G^{\prime \prime}\left(\omega, T_{0}\right) .
$$

Here, $T$ is the temperature, $T_{0}$ denotes the reference temperature, and $a_{T}$ is the horizontal shift factor. In general, the shift factor represents the characteristics of relaxation processes of a particular polymeric material.

Dozens of formulas have been proposed in the literature to link the shift factor of master curve to the chosen reference temperature. The most recognized empirical formula is

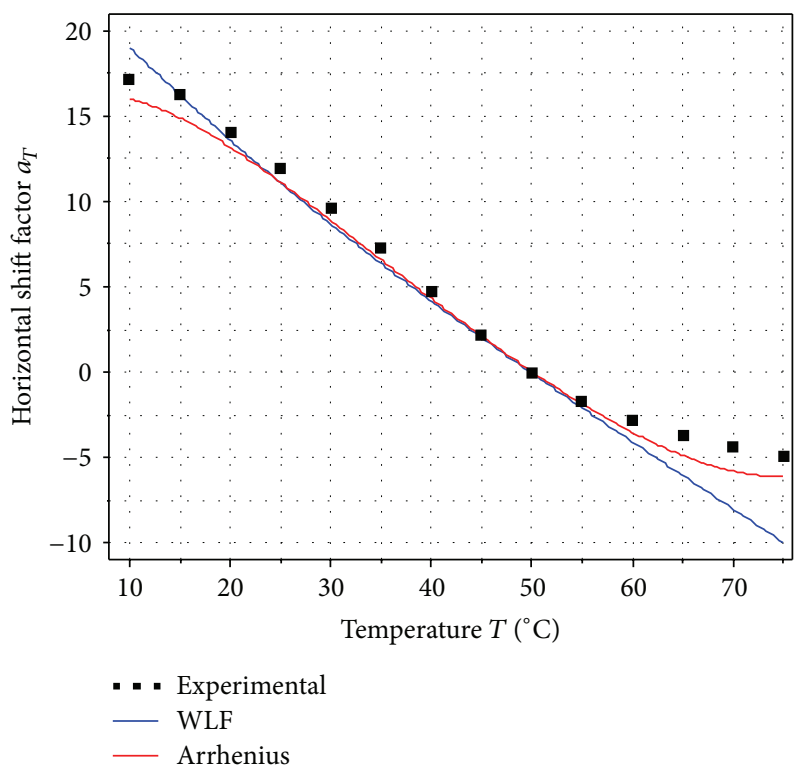

FIGURE 11: Experimental shifted data points versus temperature and comparison with WLF and Arrhenius models.

known as the Williams-Landel-Ferry (WLF) equation [14, 23, 27]:

$$
\log a_{T}=-\frac{C_{1}\left(T-T_{0}\right)}{C_{2}+\left(T-T_{0}\right)}
$$

where $C_{1}$ and $C_{2}$ are empirical constants (depending on the reference temperature) to be determined by fitting the test data of shift factor with this equation. For the data shown in Figure 10, the values of these constants have been obtained, $C_{1}=52$ and $C_{2}=149$.

The second widely considered theoretical equation for the shift factor is known as the Arrhenius model [14, 23, 27]:

$$
\log a_{T}=\frac{E}{R}\left(\frac{1}{T}-\frac{1}{T_{0}}\right) .
$$

Here, $R$ denotes the ideal gas constant $(R=8.314 \mathrm{~J} / \mathrm{moL} / \mathrm{K})$ and $E$ (measured in $\mathrm{J} / \mathrm{moL}$ ) is the activation energy.

A comparison with experimental values of the shift factor shows that the WLF equation fits data reasonably well except at the lowest and highest temperature values (Figure 11). As it may be seen in the same figure, the Arrhenius model also fails to represent data in the entire temperature range. These observations support the earlier conclusion that the SMP Estane may be considered as the thermorheologically simple material only in limited range of frequencies.

A smooth master curve for the storage or loss modulus of the tested polymer may be constructed within the more general concept of thermorheologically complex (TRC) materials $[23,26]$. However, this concept involves the necessity of introducing the vertical shift factor besides the horizontal one and this remains a contested issue in the literature [26]. 


\section{Conclusions}

This work is complementary to the parallel study by Mogharebi et al. [17] and represents a step forward in the characterization of a little known SMP Estane. It is worth pointing out the following results.

(i) The linear viscoelastic range (LVR) determined by amplitude sweep tests run at different temperatures proves that this polymer exhibits the linear behavior in both glassy and rubbery phase in a far wider range than it is usually suggested for the DMTA measurements.

(ii) The temperature dependency of the storage and loss moduli determined from temperature sweep tests shows the characteristic behavior typical for thermoplastics.

(iii) The commonly used Williams-Landel-Ferry equation and Arrhenius model to describe the temperatureand time-dependent behavior of polymers are not strictly applicable for the SMP Estane.

(iv) The master curves built up by means of a procedure based on the time-temperature superposition principle show that the tested polymer may be considered as rheological simple only in limited time range.

The primary aim of the related work [17] was to investigate the functional properties of the same type of SMP through the standard shape memory thermomechanical cycles and thermal properties using DSC and DMTA technique. Some properties of the Estane measured in this paper and in [17] agree fairly well, at least qualitatively. For example, the glass transition temperature determined in [17] by DSC and DMTA methods (around $328 \mathrm{~K}$ ) coincides with the values that may be read off from the pick of the loss factor data presented in Figure 4 (around $54^{\circ} \mathrm{C}=327 \mathrm{~K}$ ). However, the data presented in [17] are not directly comparable with the data presented in this paper for two reasons:

(1) in [17], the tested SMP (designation "Estane ETE 75DT3 NAT022") was obtained from Lubrizol Corporation as a granulate and samples have been produced by the injection molding process, while in our study the same SMP was received from Lubrizol in the form of plates with unknown processing (Figure 1);

(2) DMTA data presented here and in [17] were obtained using different testing rigs (MCR-301 from Anton Paar and Eplexor 500N from Gabo, resp.) and different deformation modes (torsion deformation giving the shear moduli $G^{\prime}$ and $G^{\prime \prime}$ and three-point bending deformation giving the tension moduli $E^{\prime}$ and $E^{\prime \prime}$, resp.). As discussed in [22], the "exact" comparison of the shear moduli with the tension moduli requires the dynamic Poisson ratio. Unfortunately, none of the used test rigs measures the Poisson ratio. However, even a very rough comparison based on the gross assumption that $E^{\prime}=3 G^{\prime}$ gives a reasonable good coincidence of the two data.
When comparing the experimental results obtained in this work for the Estane with partial data published in the literature for other types of SMPs, the following aspects may be noted.

(i) The shift in $\tan \delta$ peak to a higher temperature with the increase of frequency of the scan for the Estane (Figure 7(b)) is smaller than that obtained by Klesa [20] for the Veriflex and the Tecoflex given in [22]. This implies that the glass transition temperature increases with the increase of frequency for all these SMPs (as expected) but to a lesser degree for the Estane.

(ii) The storage and loss moduli as well as the loss factor of the Estane measured at isochronal conditions ( $f=$ $1 \mathrm{~Hz}$ ) for the heating rates $\beta=0.5,1$, and $2^{\circ} \mathrm{C} / \mathrm{min}$ show only small variations in the tested temperature range $-5-150^{\circ} \mathrm{C}$ (Figure 7 ). It then follows that the glass transition behavior does not appreciably alter with a change in heating rate in the tested range. A similar influence of the heating rates was also observed for the SMP tested by Yakacki et al. [28].

It may be briefly stated that the results of this work provide the experimental basis for the study of structure-property relationships and shape memory properties of the Estane.

\section{Conflict of Interests}

The authors declare that there is no conflict of interests regarding the publication of this paper.

\section{References}

[1] A. Lendlein and S. Kelch, "Shape-memory polymers," Angewandte Chemie -International Edition, vol. 41, no. 12, pp. 20352057, 2002.

[2] C. Liu, H. Qin, and P. T. Mather, "Review of progress in shapememory polymers," Journal of Materials Chemistry, vol. 17, no. 16, pp. 1543-1558, 2007.

[3] I. A. Rousseau, "Challenges of shape memory polymers: a review of the progress toward overcoming SMP's limitations," Polymer Engineering and Science, vol. 48, no. 11, pp. 2075-2089, 2008.

[4] J. Leng, H. Lu, Y. Liu, W. M. Huang, and S. Du, "Shape-memory polymers-a class of novel smart materials," MRS Bulletin, vol. 34 , no. 11, pp. 848-855, 2009.

[5] P. T. Mather, X. Luo, and I. A. Rousseau, "Shape memory polymer research," Annual Review of Materials Research, vol. 39, pp. 445-471, 2009.

[6] W. Wagermaier, K. Kratz, M. Heuchel, and A. Lendlein, "Characterization methods for shape-memory polymers," Advances in Polymer Science, vol. 226, no. 1, pp. 97-145, 2010.

[7] M. Heuchel, J. Cui, K. Kratz, H. Kosmella, and A. Lendlein, "Relaxation based modeling of tunable shape recovery kinetics observed under isothermal conditions for amorphous shapememory polymers," Polymer, vol. 51, no. 26, pp. 6212-6218, 2010.

[8] C. Schmidt, A. M. S. Chowdhury, K. Neuking, and G. Eggeler, "Stress-strain behavior of shape memory polymers by $1 \mathrm{WE}$ method: application to tecoflex," Journal of Macromolecular Science A, vol. 48, no. 3, pp. 204-210, 2011. 
[9] A. M. S. Chowdhury, C. Schmidt, K. Neuking, and G. Eggeler, "Comparative studies on thermomechanical behavior of veriflex, a shape memory polymer, for a low strain $\left(\varepsilon_{\mathrm{m}}=70 \%\right)$ : laser experiments," Journal of Macromolecular Science A, vol. 48, no. 9, pp. 707-712, 2011.

[10] M. Heuchel, T. Sauter, K. Kratz, and A. Lendlein, "Thermally induced shape-memory effects in polymers: quantification and related modeling approaches," Journal of Polymer Science B, vol. 51, no. 8, pp. 621-637, 2013.

[11] T. Sauter, M. Heuchel, K. Kratz, and A. Lendlein, "Quantifying the shape-memory effect of polymers by cyclic thermomechanical tests," Polymer Reviews, vol. 53, no. 1, pp. 6-40, 2013.

[12] T. D. Nguyen, "Modeling shape-memory behavior of polymers," Polymer Reviews, vol. 53, no. 1, pp. 130-152, 2013.

[13] N. W. Tschoegl, The Phenomenological Theory of Linear Viscoelastic Behavior, Springer, Berlin, Germany, 1989.

[14] J. Ferry, Viscoelastic Properties of Polymers, John Wiley \& Sons, New York, NY, USA, 3rd edition, 1980.

[15] K. P. Menard, Dynamic Mechanical Analysis: A Practical Introduction, CRC Press, Washington, DC, USA, 1999.

[16] R. Xiao, J. Choi, N. Lakhera, C. M. Yakacki, C. P. Frick, and T. D. Nguyen, "Modeling the glass transition of amorphous networks for shape-memory behavior," Journal of Mechanics and Physics of Solids, vol. 61, no. 7, pp. 1612-1635, 2013.

[17] S. Mogharebi, R. Kazakevičiūtè-Makovska, H. Steeb, G. Eggeler, and K. Neuking, "On the cyclic material stability of shape memory polymer estane," Materialwissenschaft und Werkstofftechnik, vol. 44, no. 6, pp. 521-526, 2013.

[18] Anton Paar Germany GmbH, Physica MCR: The Modular Rheometer Series Anton Paar, 2006.

[19] Anton Paar Germany GmbH, Rheoplus Software, Software Version: 3.0x, 2006.

[20] J. Klesa, "Experimental evaluation of the properties of Veriflex shape memory polymer," in Konference Studentské Tvurci Činnosti (STC '09), 2009.

[21] Z. Xia, H. J. Sue, A. J. Hsieh, and J. W. L. Huang, "Dynamic mechanical behavior of oriented semicrystalline polyethylene terephthalate," Journal of Polymer Science B, vol. 39, no. 12, pp. 1394-1403, 2001.

[22] R. Kazakevičiūtè-Makovska, S. Mogharebi, H. Steeb, G. Eggeler, and K. Neuking, "A critical assessment of experimental methods for determining the dynamic mechanical characteristics of shape memory polymers," Advanced Engineering Materials, vol. 15, no. 8, pp. 732-739, 2013.

[23] J. Dealy and D. Plazek, "Time-temperature superposition-a users guide," Rheology Bulletin, vol. 78, no. 2, pp. 16-31, 2009.

[24] A. J. Levine and S. T. Milner, "Star polymers and the failure of time-temperature superposition," Macromolecules, vol. 31, no. 24, pp. 8623-8637, 1998.

[25] O. Starkova and A. Aniskevich, "Limits of linear viscoelastic behavior of polymers," Mechanics of Time-Dependent Materials, vol. 11, no. 2, pp. 111-126, 2007.

[26] N. A. Hardikar, S. Bobba, and R. Jha, "Applicability of time temperature superposition principle to an immiscible blend of polyphenyleneoxide and polyamide," Journal of Polymer Engineering, vol. 31, no. 2-3, pp. 223-236, 2011.

[27] Y. F. Shutilin, "Use of the Williams-Landel-Ferry and Arrhenius equations in describing the relaxational properties of polymers and polymer homologues," Polymer Science U.S.S.R., vol. 33, no. 1, pp. 119-127, 1991.
[28] C. M. Yakacki, S. Willis, C. Luders, and K. Gall, "Deformation limits in shape-memory polymers," Advanced Engineering Materials, vol. 10, no. 1-2, pp. 112-119, 2008. 

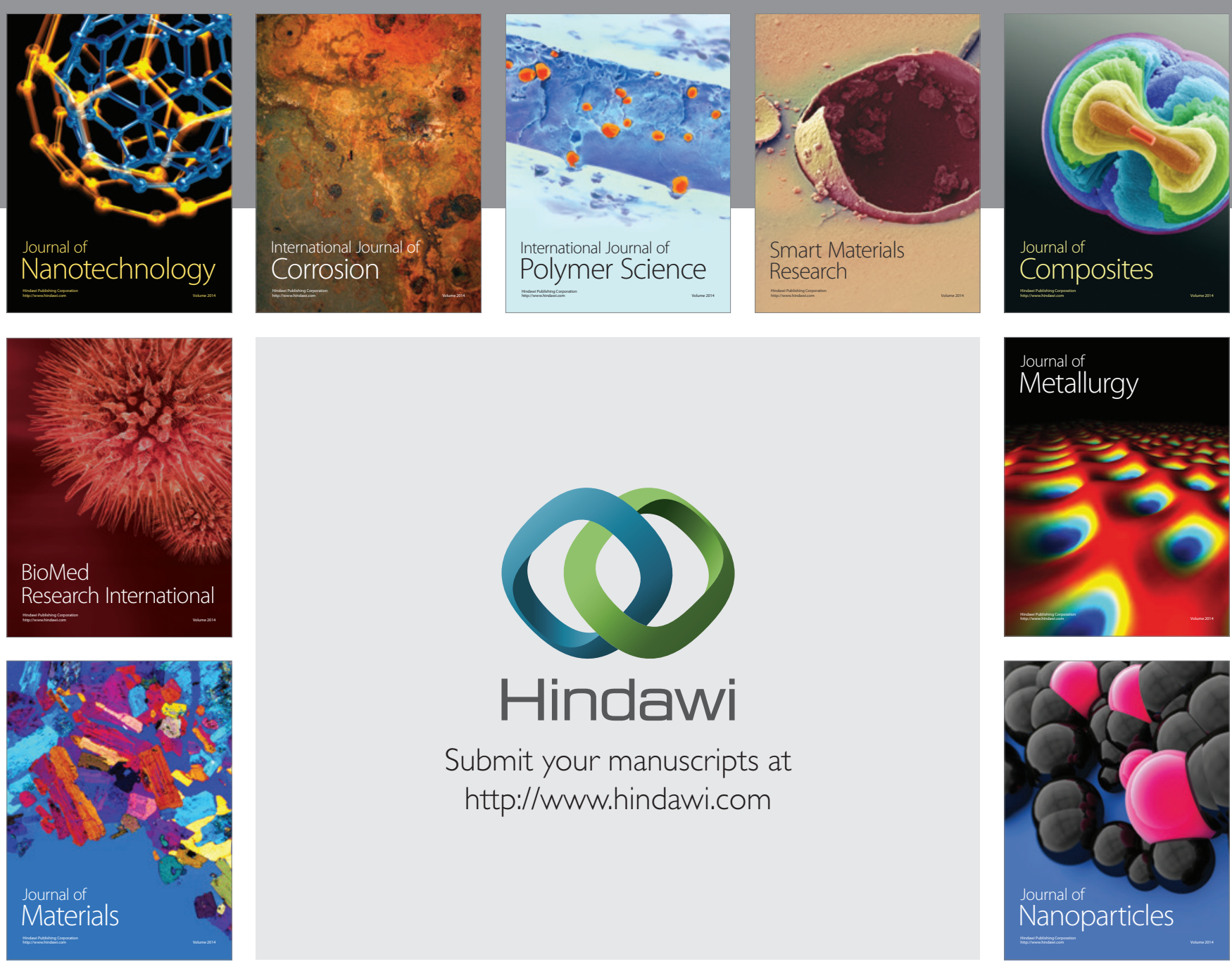

Submit your manuscripts at http://www.hindawi.com
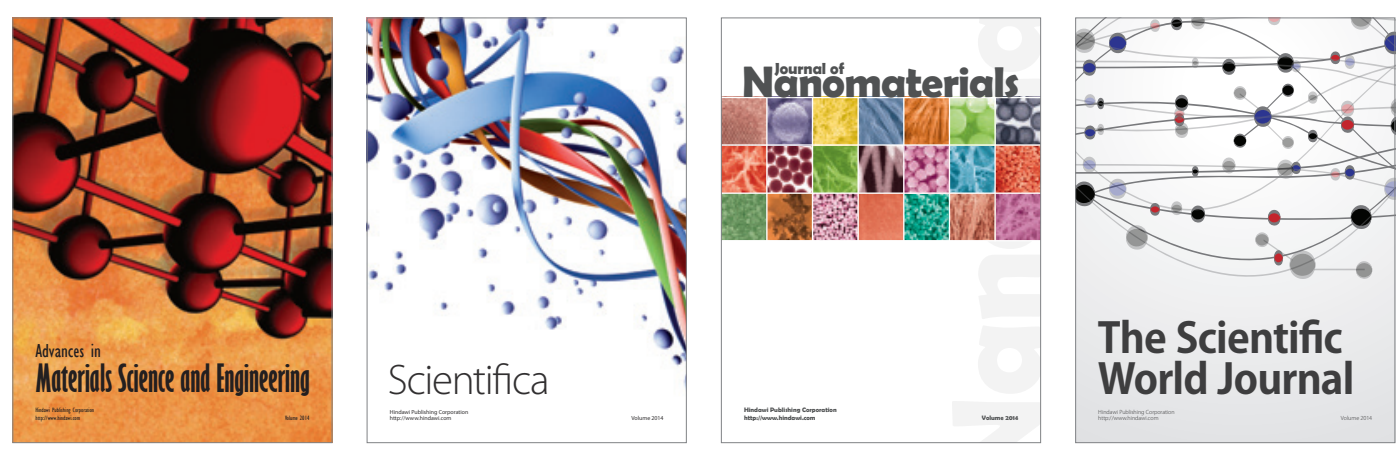

\section{The Scientific World Journal}
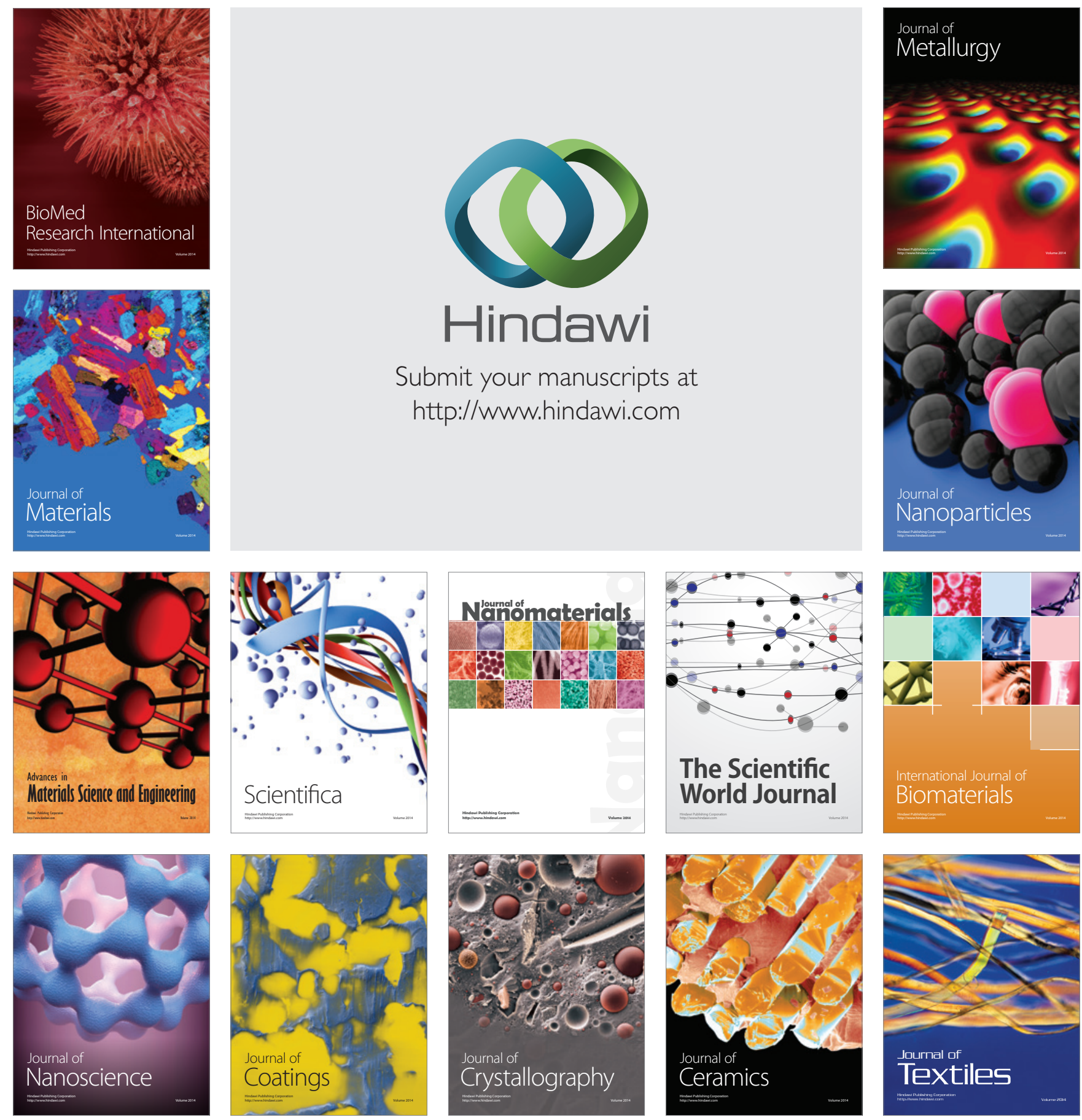